\title{
ENGINEERED NANO-MATERIALS FOR ENERGY AND THEIR POTENTIAL IMPACT ON ACTUAL ENVIRONMENTAL ISSUES
}

\author{
Liviu Popa-Simil 1 $ه$ \\ ${ }^{1}$ PhD, LAAS, Los Alamos, NM 87544, USA.
}

Received 17 October 2021

Accepted 17 November 2021

Published 30 November 2021

\section{CorrespondingAuthor}

Liviu Popa-Simil, laaos@laaos.org DOI

10.29121/granthaalayah.v9.i11.2021 .4374

Funding: This research received no specific grant from any funding agency in the public, commercial, or not-for-profit sectors.

Copyright: (C) 2021 The Author(s). This is an open access article distributed under the terms of the Creative Commons Attribution License, which permits unrestricted use, distribution, and reproduction in any medium, provided the original author and source are credited.

\section{ABSTRAC'T}

It is known for more than 2000 years that Damascus swords' performances were not possible without the existence and usage of the "Damascus steel", the first man made nano-hetero structural material that generalized is clearly showing that materials determine ultimate properties of the objects that made of. The actual world energy is manly based carbon emission materials, as coal, heavy oil, methane gas, with negative environment impact. Solar, wind and geothermal energy have also a negative impact on environment and have to be smartly used to minimize it. Nuclear energy, has lower $\mathrm{CO} 2$ emission, but because it is in its infancy it is complex, expensive and raises security and proliferation issues, has the potential for large scale accidents, and generates difficulties in dealing with waste fuel dispositioning.

The novel developed families of engineered nano-materials, eliminate all the drawbacks of the actual nuclear power, rendering it among the most efficient and environmentally friendly energy source. We learned from the global warming that the amount of energy man can produce on Earth is limited at $0.1 \%$ of sun delivered on Earth energy of $170 \mathrm{PW}$, which is of $200 \mathrm{TW}$, about 100 times more than today if it is chemical pollution free. Thermal pollution remains in place, therefore the upper clean power limit acceptable for the planet is at about $100 \mathrm{TW}$.

The novel nuclear materials were developed in 6 families, each of them bringing in harmony a nuclear agent active inside that material as:

- Micro-hetero structures, generally called "cer-Liq-Mesh", that self-separates the fission products from the nuclear fuel and minimizes their fuel damage, allowing breed \&burn to near perfect burning;

- Nano-hetero structures generically called "CIci", that form a super-capacitor, charged by nuclear energy and directly discharged as electricity;

- Nano-clustered structure that enhances self-separation of transmutation products;

- Fractal immiscible materials with radiation damage self-repairing capabilities eliminating the need for re-cladding in near perfect burning structures

- Nano-structures with active NEMS used as fast control of nuclear reactivity by guiding neutrons in desired directions or ultralight shielding for mobile reactors.

- Nano-structures that create active-quantum-nuclear-environment for long range nuclear reactions control by using quantum states entanglement and collective quantum states control.

The use of these advanced materials in future nuclear energy related application will render a high efficiency, minimal nuclear waste, and optimal nuclear fuel cycle, delivering the needed planetary clean energy at will for the next 10,000 years.

Keywords: Engineered, Materials, Environmental, Energy, Issues

\section{INTRODUCTION}

The nuclear power is receding due to many problems it encountered which rendered it less competitive with other energy production methods, while on its military aspects it is proliferating and thriving due to the novel requirements triggered by hypersonic delivering vectors John (2018) From the military point 
of view of primary application as a destructive device the explosive systems reached their maturation Lara (2016), but still remains a highly pollutant weapon where less than $5 \%$ of the fissile fuel is burned up and with power delivery capability well over strategical-tactical usefulness. As nuclear power world is multipolar MAD (Mutual Assured Destruction) maintains the peace, but recent pushes from ignorant politicians nourished the hope for old "blitz Krieg" concept, using hypersonic carriers and stealth nuclear submarines, that is a destabilizing falsehood. On UN arena NPT (Nuclear Non-Proliferation treaty) is losing ground, mainly under US effort to develop novel weaponry and international aggressiveness.

From the point of view of "Atoms for Peace", civil nuclear power is losing ground Staub (1967), becoming less and less competitive in front of novel power production technologies due to multiple reasons:

- Nuclear power is more expensive both as initial investment, maintenance costs and production;

- Nuclear power is more complex, requiring highly educated and trained personnel

- $\quad$ The safety measures needed to be implemented and applied are laborious and costly

- The security and nuclear non-proliferation are mainly achieved by G3 philosophy: Gates, Guards with Guns, which make the presence in an area a real factor of discomfort

- Nuclear hazard and accidents and anti-nuclear propaganda fuelled by ecological and fosil fuel industry made the new nuclear reactor construction tumble;

- Nuclear waste remains a big problem, currently unsolved because there is no easy, clean solution Sakis et al. (2005).

All these issues contributed to the fact that after 1986 Chernobyl accidents the number of reactors to remain steady at about 440, and after Fukushima accident by 2011 some nations as Germany to opt-out, now confronting with acute energy issues, being bound to Russian methane gas or American LPG.

The decay of the active nuclear reactor number started by 2012, by not building new nuclear reactors with the exception of China, India, Brazil and due to the fact, the nuclear fleet is old, and inspire iffy life extensions granted by NRC in the US nothing else have been done. In spite of moribund nuclear power, the guilt of nuclear people starts talking about "Nuclear Renaissance", based on wishful thinking, ignoring the fact that the issues mentioned above have to be solved first, some see the escape by promoting "Small, Modular Nuclear Reactors", hoping to trick the public negative opinion, making them believe that smaller may be safer and more secure, which is another falsehood.

It turns to be true, the Oren Harari famous saying: "Electric light is NOT the result of continuous enhancements in candle technology", applied in Nuclear Power, where the guild is among the most conservative one, and prefers little to none evolutionary changes, at high cost and low risk and reward, just executing a smooth spending with known, most preferred and connected people, and this practice in the US is called "business as usual"? As a consequence, the openness to new technologies is mainly on paper, but considering the national response to covid-19, where the propagation is done by nano-size aerosols, it is clear that American minds are not cabled to effectively understand the nano-technologies; in spite they are prone to use to abuse the word "nano". 


\section{ISSUES OF THE ACTUAL NUCLEAR POWER}

As it is shown in Figure 1 upper-right side Nuclear Power MWh is expensive, making it less competitive.

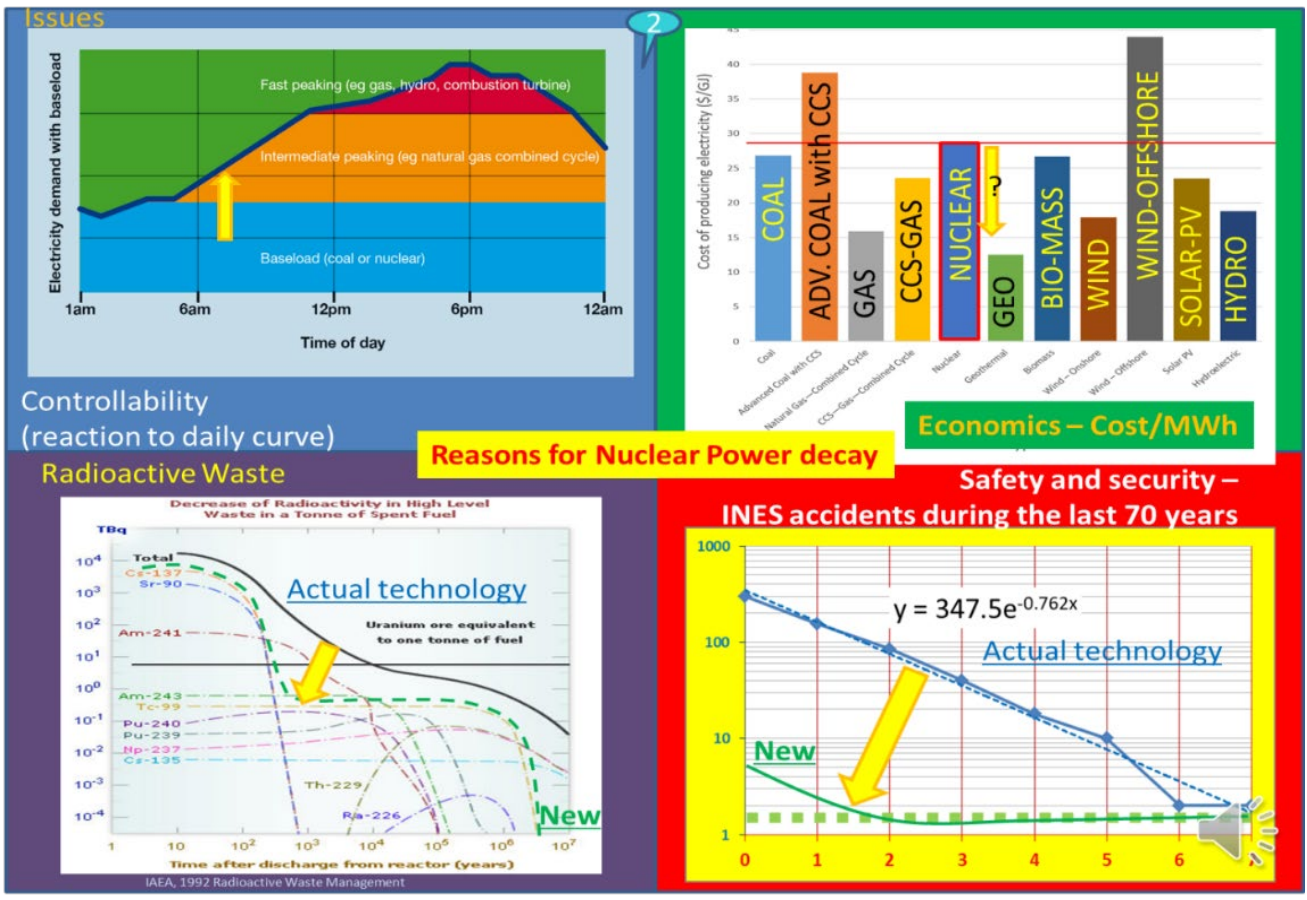

Figure 1 Issues of the present nuclear power production

In national energetic systems nuclear power is used as base line energy, because steering a nuclear reactor and making it follow the daily demand curve is very difficult, due to particularities of the actual criticality control system that uses mainly delayed neutrons the control reactivity. The actual technology has a low burnup and high dilution of fission products into nuclear waste fuel, therefore high amount of waste has to be stored, and with minor actinides not extracted it remains a radioactive hazard up to a million years, as seen in lower-left chart. Safety inside nuclear reactor is another issue, and the lower-right chart is showing the number of events, classified as incidents under INES $<4$ and accidents above, but in spite nuclear is safer than other power production technology by more than 1 order of magnitude, the capability of producing INES=7 accidents every 25 years is scary. Now, due to nuclear fleet reduction the next INES7 accident may happen after more than 35 years, or not happened at all because by 2050 very few reactors from the actual fleet will remain operational.

\subsection{REASONS BEHIND THE ACTUAL NUCLEAR POWER DRAWBACKS}

It is known that inside a nuclear reactor there are several processes acting simultaneous that have to be in harmony with the materials hosting them, but they are not Popa-Simil (2007). 


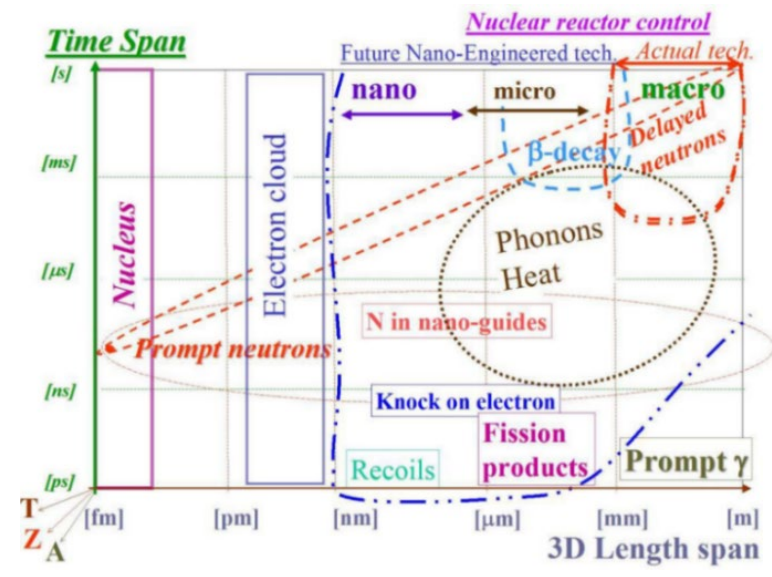

Figure 2 multi-scale multi-dimension D-T plot of nuclear processes

AS one may see in Figure 2, in a multi-dimension (L; T; Z; A..), with scale from pico-second and femto-meter to unit (s;m) the only entity we have a correct spacetime grab on it are the delayed neutrons, as the double-red-dotted-line shows for the "Actual technology". With some approximation $\beta$-decay that is related to fission products is contained. In order to improve the process inside and to eliminate the drawbacks of the actual technology, one needs to bring harmony and develop means to deal with fission products, knock-on electrons, prompt neutrons, recoils, gamma and beta decay Popa-Simil (2015).

\section{HISTORY OF MICRO-NANO-NUCLEAR MATERIALS DEVELOPMENT}

The studies related to "special nuclear fuels" started in late 1970s as an initiative of Prof. Ionel Purica, Ursu et al. (1978), Schmidt George (2006) with government funding. By that year we looked mainly at direct nuclear energy conversion in electricity, using a hybrid system, to obtain multipactor electron multiplication in plasma discharge. Later by 1980 micro-structures have been developed and some nano-structures extending the scope of the research to transmutation products, in fuel breeding applications and energy conversion.

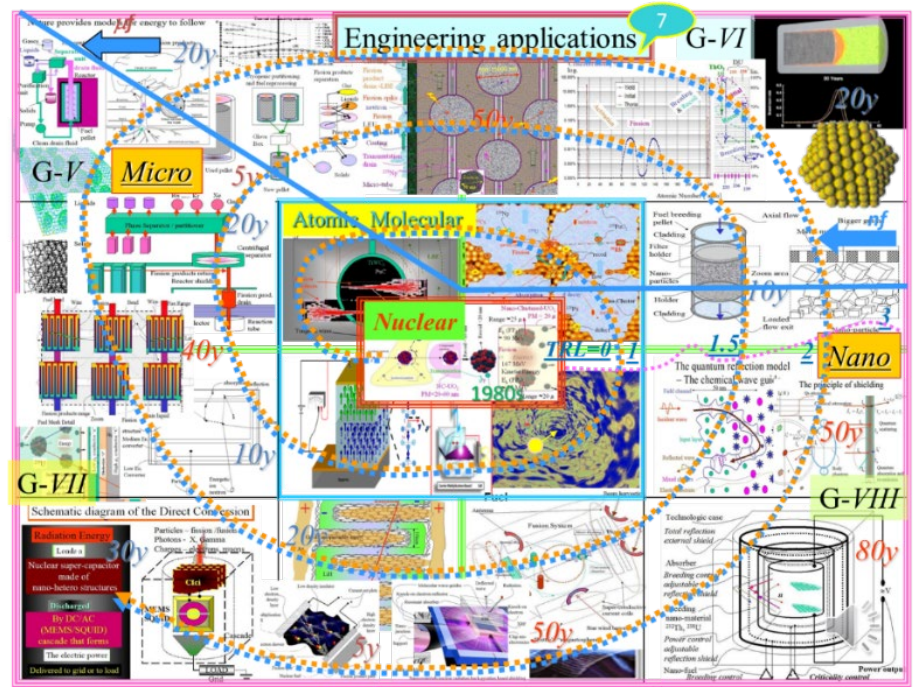

Figure 3 Micro-nano-hetero nuclear materials spiral of evolution 
As one may see in Figure 3, it started with the study of nuclear reactions at sub $\mathrm{nm}$ level, then develop nano materials from atomic and molecular level to mezzoscale, reaching TRL=1. After another 10-11 years the first structures have been envisaged, and develop test models. After year 2000 computer simulations have been made, helping to better understand empirical experiments of the past and Popa-Simil (2007) patenting the products, reaching TRL=2. From here more simulations and application diversification have been made, reaching by 2020 TRL=3, Popa-Simil (2015). In Figure 3, G and roman number means the nuclear reactor generation that may be produced from that structure and yeas nearby represent the estimated time to maturation of the technology. The potential applications are also presented as ideograms.

\section{MICRO-HETERO FUEL SELF-SEPARATES FISSION PRODUCTS}

The first and easier to produce structure is a heterogeneous micro-structure able to put in harmony the fission products with the lattice they are stopping in. Fission products are the main energy carriers, sharing about $170 \mathrm{MeV}$ out of 203 $\mathrm{MeV} 235 \mathrm{U}$ releases during fission Olander (1976). During their slowdown process inside fuel lattice, they behave like medium mass accelerated ions. From the interaction with matter behaviour, we distinguish 3 main zones, as the fission spot vicinity, of "nest", few lattice constants thick, then the stopping by ionization and finally the recoil zone, also called "Bragg peak". It is known that fission products (FPs) cover a wide range of isotopes, symmetrically distributed around the middle mass $(A=118$, for $235 \mathrm{U}+1 \mathrm{n})$ as one may see in Figure 4, lower-right side for various nuclear fuels as $235 \mathrm{U}, 239 \mathrm{Pu}$ or mixed fuel. The measurement has been made at some moment in time after fission took place, using, thermal, epithermal and fast neutrons. In reality on the isotope map, one may see that after fission the light and heavy FP arrives in neutron deficitary zone, shown in red, with probability of occurrence given by the colour of the dot and by a sequence of $\beta$ decays reach the stability. The lifetime of each isotope is given by the colour of the square.

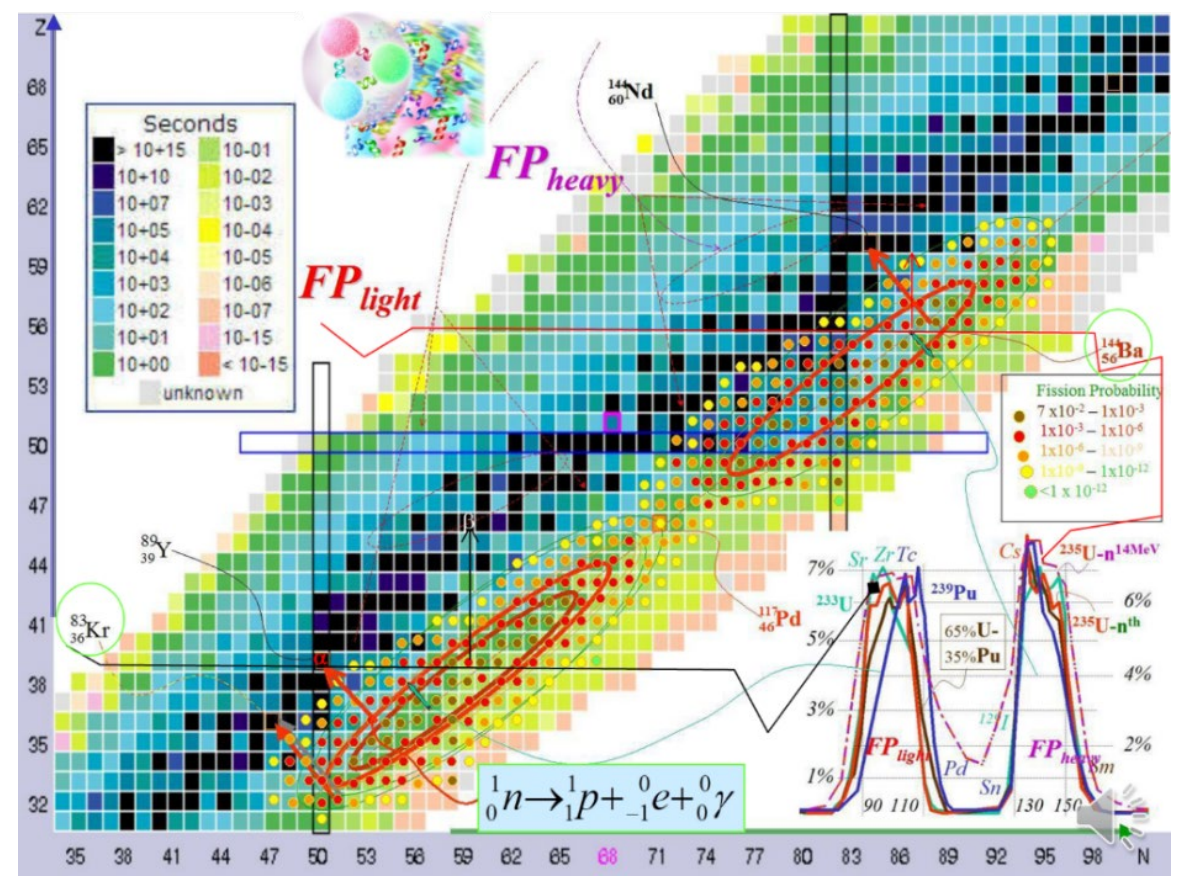

Figure 4 Fission Products distribution, lifetime and decay 
One has to understand that from creation to stability most of isotope's morph in more than half of Mendeleev table groups, changing its chemical properties, and by this is chemically destabilizing the fuel lattice. The energy stored in FPs is $>8 \mathrm{MeV}$, and it is gradually released at each $\beta$ decay and another $8 \mathrm{MeV}$ released in neutrinos energy, that is mainly lost in space, due to the ultra-small neutrino interaction cross section. At modelling, it is important to understand the nucleus structure as a soup of quarks and not as a bunch of two type balls: neutrons (udd) and protons (udu). In a nuclear fuel the FP are stopping in about 20-30 $\mu \mathrm{m}$, while on the last $2 \mu \mathrm{m}$ creates a Bragg peak, that looks like a micro-cavitation, the lattice state becomes a micro-plasma that cools down in ps the lattice partial recovering but leaving behind many defects as a result of dislocations. During this process the lattice micro-grains become fragmented and inter-boundary spaces are created accumulating fission products and changing material properties Weber (1956). The problems created by fission products in the fuel are cracks, swallowing, thermal conduction deterioration, and fission products migration towards cladding and outside, taking out of use early the fuel pellet. By design, in ceramic fuels, as Urania, the canter of the fuel pellet reaches high temperatures up to melting point, accompanied by material expansion and shearing stress that is a crack generator. Using metallic fuels, the melting temperature is low, and the heat flow is not improved. Carbide and Nitride fuels are more difficult to be obtained and have same sensitivity to various cooling agents and air exposure Ponomarev-Stepnoi and Usov (2000).

A solution for this problem is to consider nuclear fission reaction's kinematics, and design and build heterogeneous fuel structure which has the dimension of the fuel smaller than the effective stopping range of the fission products, which is of about $20 \mu \mathrm{m}$ in Urania, as one may see in Figure 5.

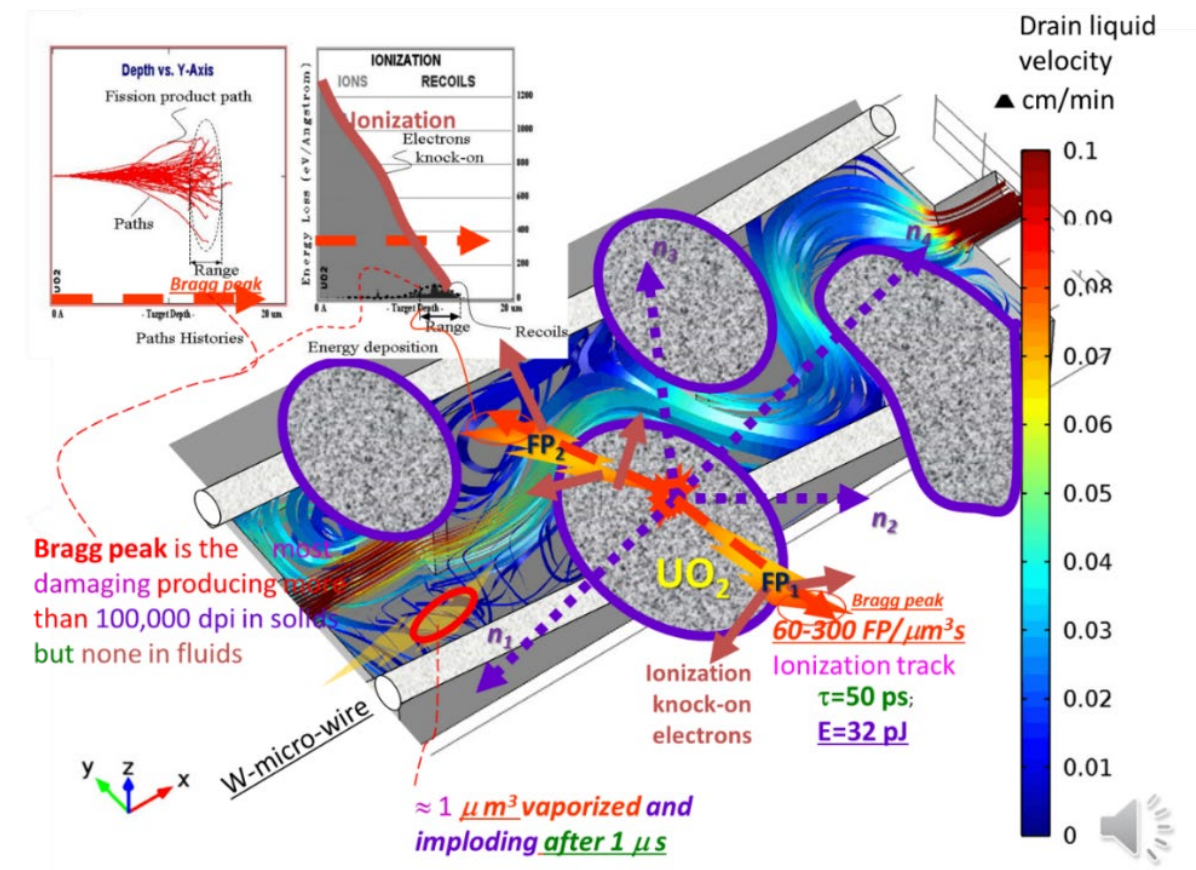

Figure 5 "Cer-Liq-Mesh", hydraulic channel and drain fluid flow

A fission is shown inside a UO2 bead (Urania) generating 4 neutrons, sharing about $8 \mathrm{MeV}$, and $2 \mathrm{FPs}$ sharing about $170 \mathrm{MeV}$, and releasing a prompt gamma flow in the fission nest, of up to $10 \mathrm{MeV}$. Fission products are released in an excited 
nuclear state and follow a decay process releasing another $8 \mathrm{MeV}$ in beta kinetic energy and another 8 in neutrino emission. The upper left charts show the range of the FPs in fuel, where the Bragg peak region is located outside the fuel bead in the drain fluid, that may be NaK or LBE, etc. In any state of aggregation Bragg peak dose, the same micro-cavity explosion followed by implosion, but liquid has no structure and after the shock wave is gone, it remains with no structural damage and only the FP at rest chemically bounding to liquid molecules. Drain fluid flows slowly, training FPs outside the active zone of nuclear reactor at open flow fuels, or if encapsulated in cladding, it will go on the upper and lower walls of the tube, being separated from the nuclear fuel. The advantage of open circuits is that the FPs are prevented from absorbing another neutrons reducing the reactivity, or as is said "poison" the reactor. Such a fuel will allow deep burnup or a breed and burn process, up to the safety limits of cladding, that is known not to be so high and allows about $10 \%-20 \%$ uranium burnout. To facilitate breed \& burn process the pellets have to be easy reprocessed and re-cladded and that is made possible by the fact that a simple extraction by sequential distillation leave the burned fuel clean and free from fission products, where compressed, to recover reactivity loss can be easy re-sealed in new cladding. Repeating this operation several times over $80 \%$ of the initial fuel is burned, with no need for enrichment and this is a revolutionary change to fuel cycle, reducing the need for mining, with modified reprocessing on site, and deep burnout.

\section{NANO-BEADED FUEL FOR ADVANCED EXTRACTION OF TRANSMUTATION PRODUCTS}

If the fission products had high energy able to take them more than $20 \mu \mathrm{m}$ away from the "nest", not the same is the situation of transmutation products where the absorbed neutron recoil energy is not enough to take the atom out of its initial location in the lattice and create an interstitial defect. In this situation, in spite the isotopic and elemental differences the transmutated nucleus will remain in its place inside the lattice Estrin and Nabarro (2007).
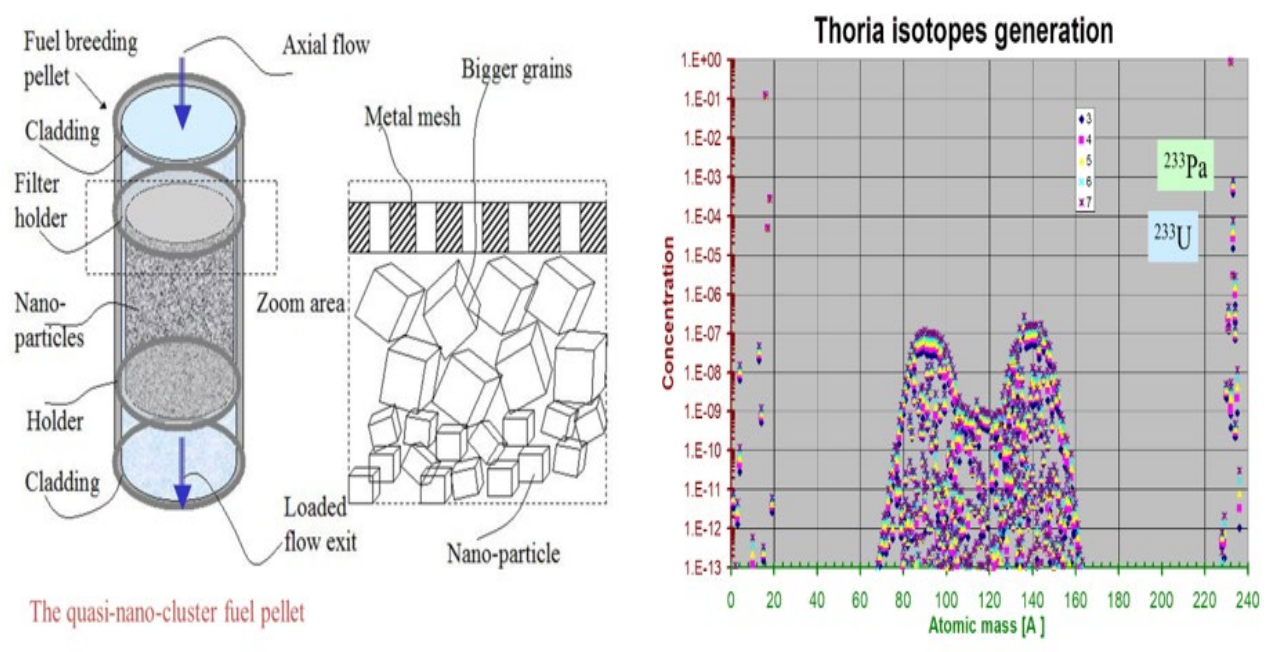

Figure 6A The nano-powder pellet schematic diagram. $\quad$ Figure 6B Thorium breeding in up to 7 days.

By mid 1980s an experiment to produce super grade 233U and 239Pu was made using depleted Urania and Thoria nano-powders into an open pellet creating a frit like device where the smaller nanobeads were placed in the middle having a 
gradual increase in particle magnitude (PM) to the ends of the tube in order to have them stabilized in place and closed with Al metal micro-foil filter with calibrated hole dimension, able to hold larger particles as seen in Figure 6A. The irradiation took place a week inside a VVRS zero power nuclear reactor and a cooling time of few days, and the extraction was made using solutions passed through nanostructure. In solution there were found various isotopes, as seen in Figure 6B. The extraction liquid contained fission products too, and recoiled 232Th together with higher concentrations of $2^{33} \mathrm{~Pa}$ and $2^{33} \mathrm{U}$, than in the case of dissolving the entire pellet, making extraction process easier and less hazardous.

The process relies on the capability of nano-clusters to have enhanced diffusion able to transport an atom acting as a defect on the boundary, from were based on affinity to the drain fluid, the transmutation atom to concentrate and be carried away by the fluid. In open systems, where drain fluid has a continuous circulation through the pellet, the transmutation products are carried away, from the reactor high flux zone, reducing the probability of them to be exposed to absorbing another neutron and fission or transmute away contaminating the desired production.

The method may be applied successfully to the entire production if radioisotopes, using nuclear reactors or accelerators. In accelerator targets, it may be also used recoil separation in corroboration with nano-cluster enhanced extraction because the incoming ion has a significant energy to overpass the Coulombian barrier, and recoils the nucleus from the lattice atom in interstices, from where the enhanced diffusion of nano-cluster puts it on grain interface.

The advantage of this method is that makes the separation and extraction process easier, with lob radiation background making complex process as Urex or Purex not needed in the context of continuum extraction and increases the production purity by early extraction from reactor hot zone.

\section{NANO-HETERO STRUCTURES FOR DIRECT NUCLEAR ENERGY CONVERSION IN ELECTRICITY}

Following the previous analysis, we have talked about kinetic energy of moving nuclear particles which in interaction with matter is deposited by interaction with atomic electron clouds and resulting showers of knock-on electrons that share the deposited energy. Number of knock-on electrons depends on neutron availability in the material and type and energy of moving particle Popa-Simil (2018).

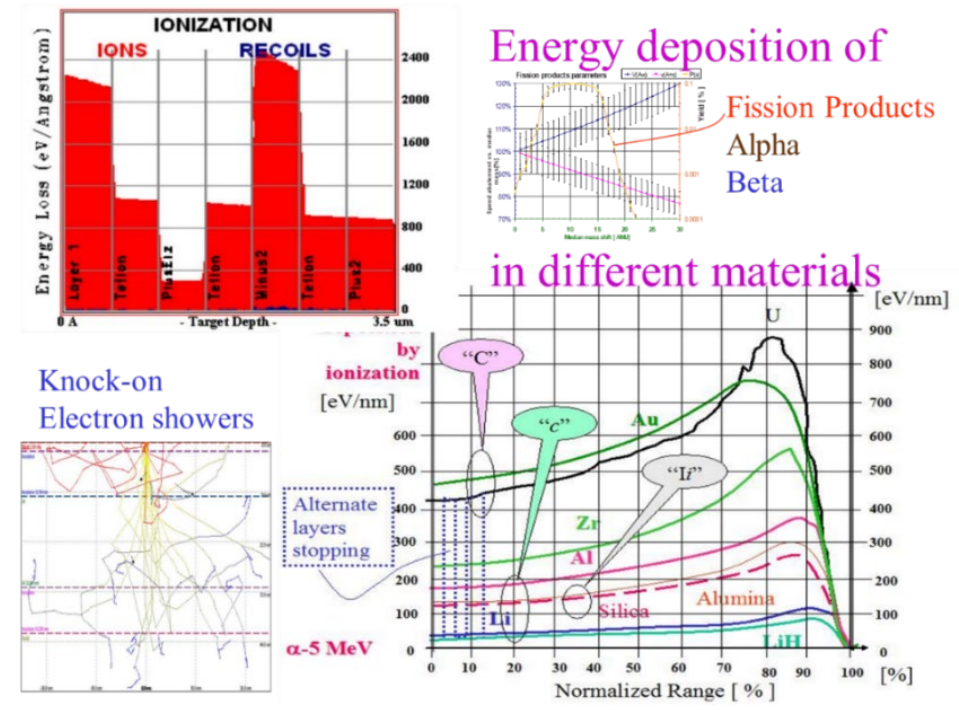

Figure 7 Knock-on electrons behaviour in materials 
It is possible to create a meta-material made of an repetition of modules made of a sequence of 4 layers, first with high electron availability, creating a big electron shower, followed by an insulator layer inside which electrons have a ballistic flight, and a conductive layer with low electron availability, that stops the shower, charging negative and does not emit a large shower along the nuclear particle path, followed by an insulator with low electron availability, generically this is called "CIci" cell, and has an approximatively thickness of about $100 \mathrm{~nm}$, being repeated by few hundred times to cover the stopping range. In Figure 7, upper left side one may see the electron energy deposition in a microstructure made of layers of $0.5 \mu \mathrm{m}$ gold, PTFE, Aluminium, PTFE repeated twice. The energy may come from fission products as seen in the upper right chart, as a function of distance from the centre mass (118 $\mathrm{amu}$, for $235 \mathrm{U}+\mathrm{n}$; or $120 \mathrm{amu}$ for $239 \mathrm{Pu}+\mathrm{n}$ ) where the light FP has higher energy than heavier partner. The chart in lower-right side shows how materials were selected based on their knock-on electron production for various portions of the stopping range. IN the lower-left side is shown the electron shower interaction with other atomic electrons creating showers of low energy high number of electrons that polarize the hetero-structure. As one may see the " $\mathrm{C}$ " layer may be made of fissile material, but structure is not robust to the recoil zone where liquidelectronics have to be used compatible with Bragg peak effects. Finally, what is made is a super-capacitor able to charge from nuclear particle energy and discharge as electricity.

In Figure 8A it is shown a planar supercapacitor structure in which the moving nuclear particle enters from the left through a "C" nano-later in red, producing a large electron shower, stopped in a "c" nano layer in blue that becomes negatively polarized. The capacitor foils are connected at plots and from there to the outside resistive load. The upper curve Vij shows the voltage between the conductive layers $\mathrm{i}$ and $\mathrm{j}$, and the lower chart shows the current Iij inside the structure. The internal dimensions are customized on particle type and its energy.

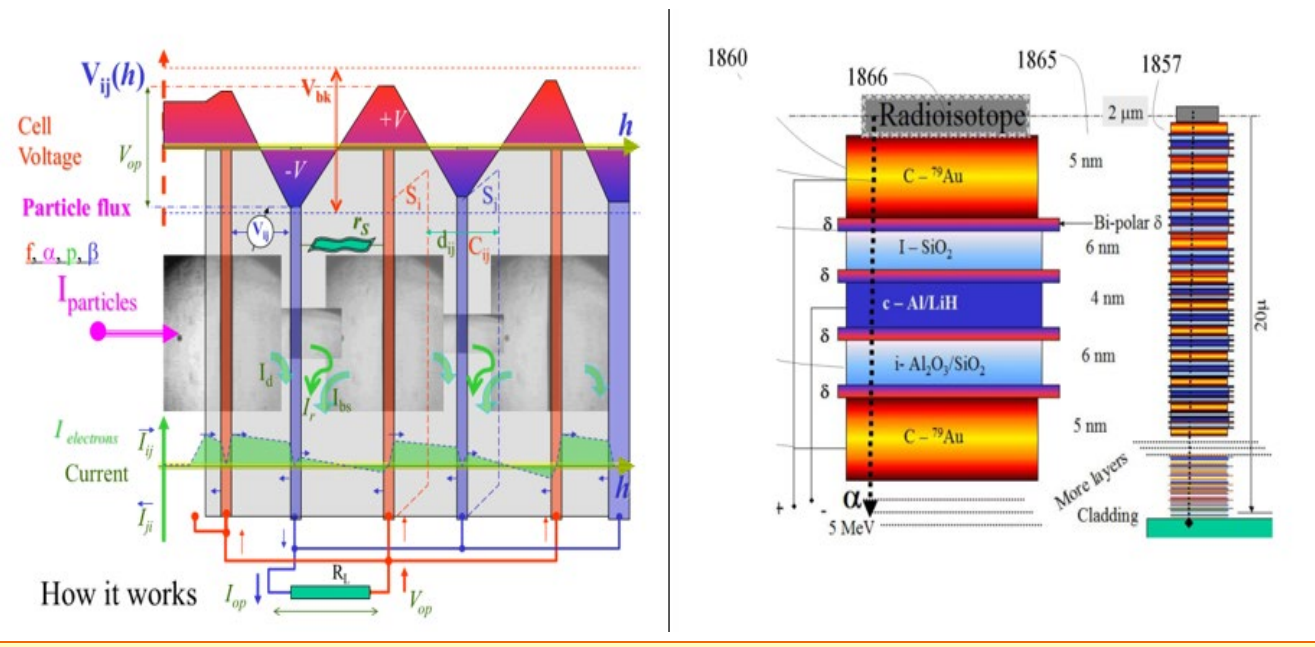

Figure 8A Planar super-capacitor operation

Figure 8B Planar structure isotopic battery

Figure 8B shows such structure using a radio isotope that is alpha or beta emitter, where the radioisotope is few microns thick and is placed between the supercapacitor foils. Typically, such a structure is about $50 \mu \mathrm{m}$ thick, and may be morphed on any structure. 


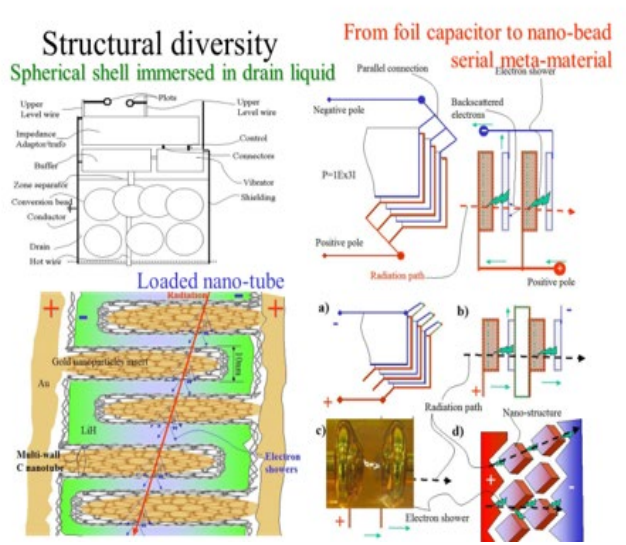

Figure 9A Variants of supercapacitors

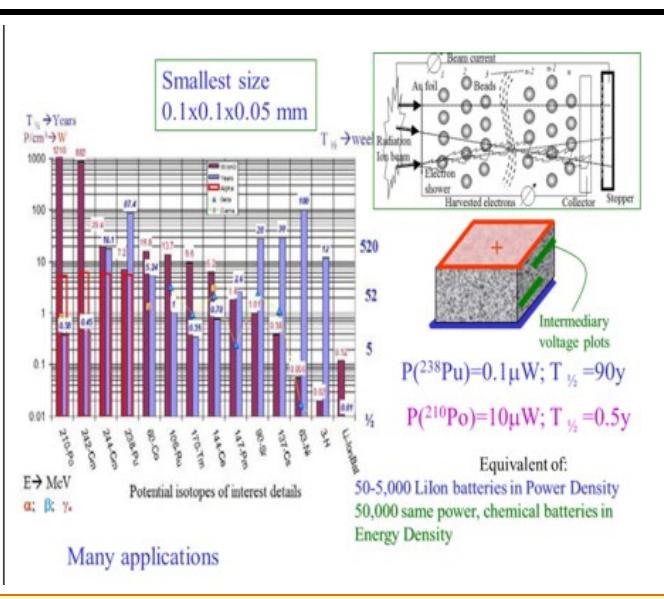

Figure 9B Isotopic batteries sources

In Figure 9A it is shown that there are several variants one may produce direct energy conversion structures, as planar structures connected in parallel delivering low voltage and high current, or in series delivering high voltage and low current, or morphed in nano-beads with the size compatible with the process, or in carbon nanotubes (CNT) immersed in LiH and fulfilled with Au or other Schmidt George (2006) electron available material. In Figure 9B, one may see an isotopic battery, made of nano-beaded meta-material, in planar geometry tuned for $\alpha$ that may have the minimum dimensions of $0.1 \times 0.1 \times 0.05 \mathrm{~mm}$ and a power level depending on the isotope load of $0.1 \mu \mathrm{W}$ for 90 years, if loaded with $238 \mathrm{Pu}$, or $10 \mu \mathrm{W}$ for $1 / 2$ years if loaded with $210 \mathrm{Po}$. The chart on the left side shows various isotopes that may be used except for $\gamma$ emitters that have large halving paths. The material may be made as a hyperbolic meta material and energy may be discharged as EM radiation, making a THz or vis. laser like device.

\section{ENGINEERED NANO STRUCTURES FOR ACTIVE RADIATION GUIDING}

Figure 10 shows a step forward some of the applications that may be achieved using nano-structures to trap radiation and guide along a nano structure path, or if a NEMS circuit is added on the path, to command it and switch the radiation path at will. The process is similar to radiation channelling developed since 1970s mainly in $\mathrm{EU}$, but a little bit more complicated due to fine details.

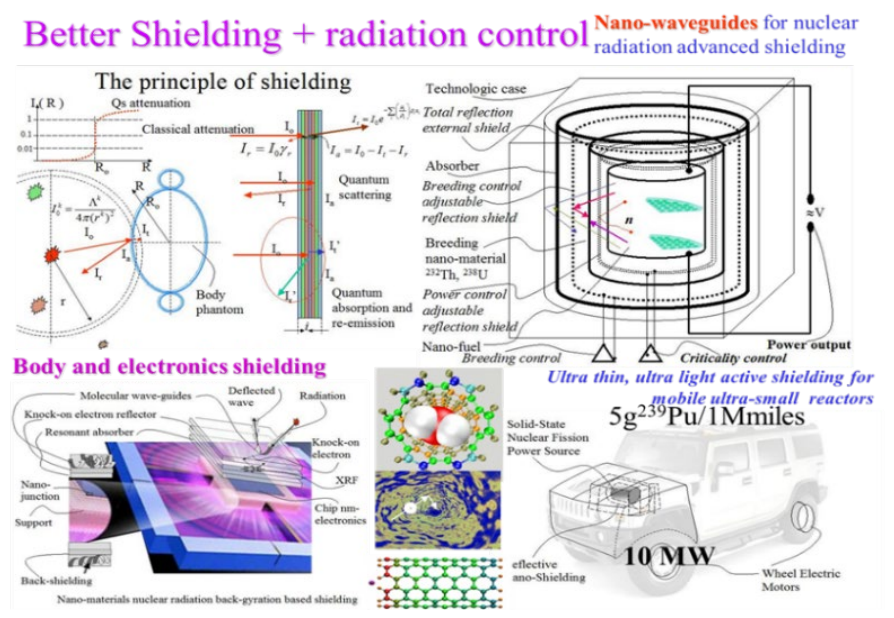

Figure 10 Structures obtained using radiation guiding in nano-structures 
In the upper-left side it is shown a shielding application that uses the 3 methods available: mass attenuation, that is currently used, called so, because no matter what isotope one may use, to attenuate a radiation above atomic resonances range, the mass will be about the same, and only dimensions will vary due to density. Another method, for narrow radiation is similar to the Mossbauer effect, and efficient scattering in $4 \pi$ may dim drastically a directed radiation. The third way is to use radiation guiding structure where a radiation behaves like a sleigh on a bob-track, being "bended", by a multitude of very little collisions at grazing angle, and being released on another direction without losing much of its energy and impulse during this multiple interaction with the nano-structure. In the lower -left side is presented a shielding for an electronic device, to prevent single event upset using the layers of guiding nano-structures to create a denial zone. On the upper-right is presented a solid-state nuclear reactor, where active guiding nano-structures were used to control its reactivity and shielding, via passive guiding. This achievement that will become mature in about 80 years from now, may make possible the development of ultra-compact solid state nuclear reactors, that may be used even at SUV level, requiring $5 \mathrm{~g}$ of $239 \mathrm{Pu}$ for $1 \mathrm{Mmiles}$, the problem is that in order to have that burnup another $5 \mathrm{~kg} 239 \mathrm{Pu}$ to be immobilized in the critical structure generically called nuclear reactor, that facilitate the burnup of overcritical material, which will be a significant deterrent for large scale application and other power systems will have to be used for general public transportation.

\section{FRACTAL NANO-HYBRID STRUCTURES WITH RADIATION- DAMAGE SELF-REPAIRING CAPABILITIES}

As we presented before, using "Cer-Liq-Mesh", micro-hetero-structures it becomes possible to perform near-perfect burning only if fuel safety conditions are met. Using actual structural materials, as stainless-steel. Moly, zircalloy, etc., maximum $10-20 \%$ of the fuel may be burned using a breed \& burn technology, because cladding is affected by radiation damage, mainly due to interaction with neutrons, and seldom with direct fission products.

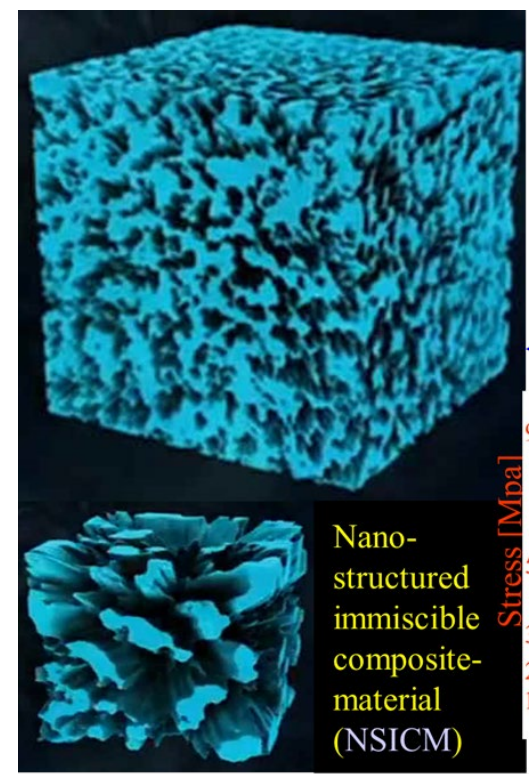

Types of nano-composite structures

Composite $\mathrm{SiC}^{\text {fiber-W,Tplating }}$

Immiscible solid solutions

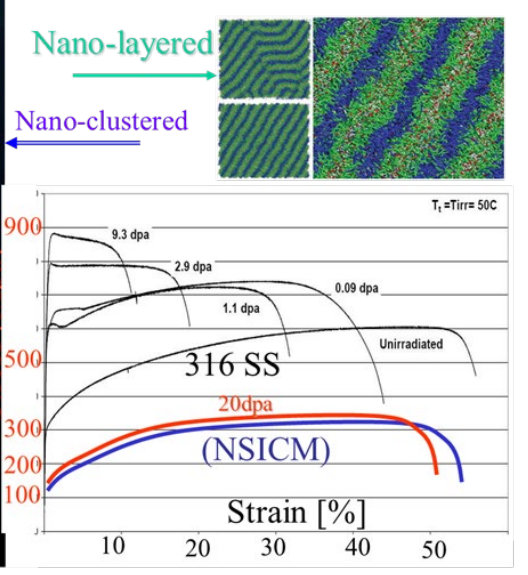

Figure 11 Fractal nano-hetero structure with radiation damage self-repairing capabilities 
The material shown in Figure 11 left-side is a solid immiscible solution, with a $3 \mathrm{D}$, fractal number 2.5 to 2.7 and dimension of the blobs of a material in the range of effective Bragg peak, mainly s sub-micron size, depending of the $\mathrm{n}$ flux type: thermal, epi-thermal, fast, etc.

These kinds of materials are weaker than the actual stainless steel, that will require thicker walls, but the interesting property is that they maintain the same properties constant with absorbed dose and equivalent dpa, because during the recovery from the Bragg peak damage it returns to about same shape.

On the right side is shown various types of composite materials, that are interesting for being use in the future inside a nuclear reactor, simply as they are made or engineered in more complex structures, controlling the isotropy of mechanical parameters on a case-based logic.

The lower-right side chart shows an example of comparative be strain-stress behaviour of 316-SS that becomes stiffer and brittle with the absorbed dpa, compared with an engineered novel material (NSICM) with little changes due to dpa level.

\section{COMPOSITE NANO-STRUCTURES FOR QUANTUM-NUCLEAR ACTIVE ENVIRONMENTS}

This is a hot, controversial subjects, which for the "monks" of physics this is meta-science, while for open-minded it is a subject that randomly occurs, and must be considered in spite it is not completely covered by the actual level of knowledge. In the race for supremacy in funding for "no results", the guild of "plasma physicists", managed to place it at the outskirts of the science labelling "cold fusion".

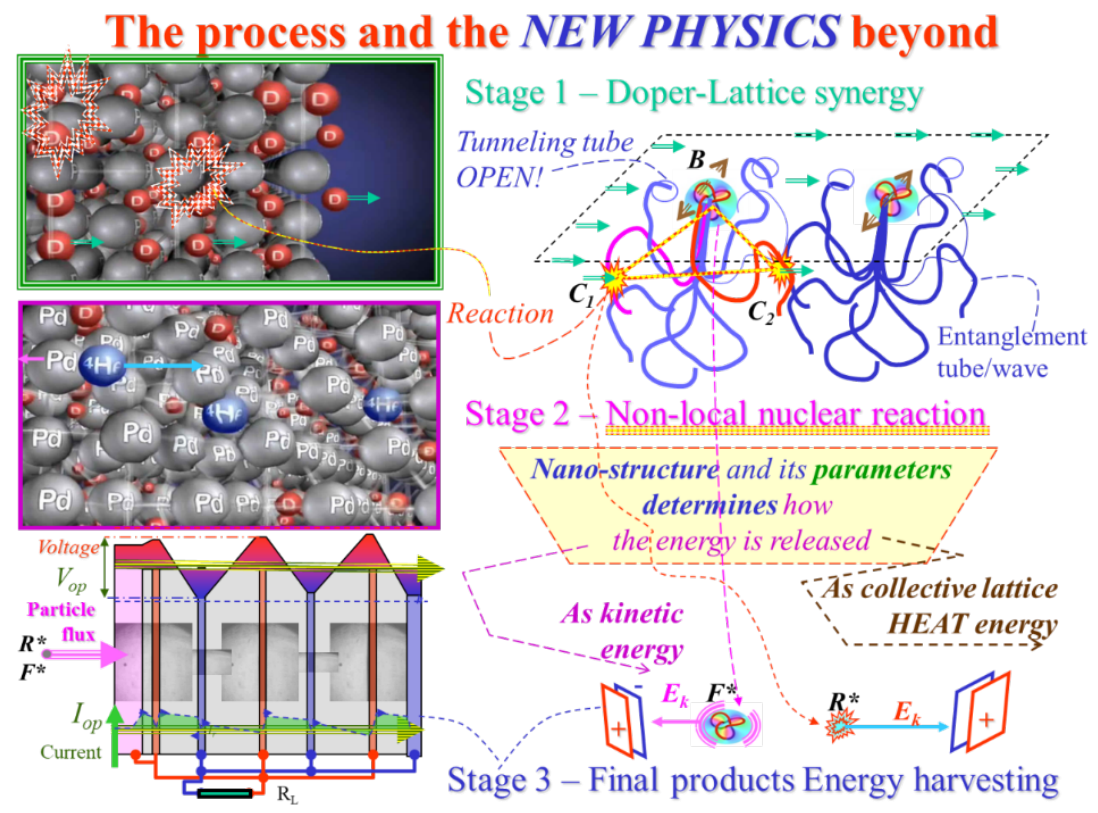

Figure 12 An "artistic view", on novel processes based on active quantum environments

The process is not new, first identified by Thomas Graham as "Some anomaly in the action of hydrogen," since 1870s, then by Frank, Herzfeld and Gopert-Mayer by 1930s, now totalling more than several thousand publications. In my view, nuclear world is strange, has so called resonance, for example the apparent size of 
$135 \mathrm{Xe}$ for thermal neutrons is $163 \mathrm{pm}$, at an atomic diameter of $432 \mathrm{pm}$, having a cross section of $2.65 \mathrm{Mbarn}$, while for fast neutrons it decays at 3 mbarn. Figure 12, shows some entanglement fibres of space that when in touch a quantum assembly is created, and quantum states transitions are possible, driving at transmutation, fusion or fission, and all may be driven by low energy resonant states, such a $\mathrm{THz}$ excitation in nano-structure. It was shown that a $\mathrm{Pd}-\mathrm{D}(80 \%)$ is more prone in reproducing anomalies than diluted states. Other materials showing anomalies are $\mathrm{H}-\mathrm{Ni}$ assemblies, and many other materials, but quantum mechanics knowledge since does not cover long range nuclear reaction with quantum state teleportation, and more progress is needed until an energy extraction application to be reliable and reproducible in any environment. The figure shows the combination of an active quantum environment generating charged particles with nano-hetero structure for direct fusion/transmutation energy conversion, as a fusion battery of the future with time to maturation of about 100y, or less if we are lucky and produce the necessary discovery. The advantage of this power source is that uses $\mathrm{H}$ isotopes has no critical mass, and has little reactive energy able to produce accidents larger than INES=4, being the most ecologic friendly future energy.

\section{ENERGY RELATED APPLICATIONS' ENVIRONMENTAL IMPACT}

The 6 families of nano-hetero-meta-materials are mainly engineered for nuclear power applications and are intended to bring in Harmony the nuclear process with the host structure. Popa Simil (2018).

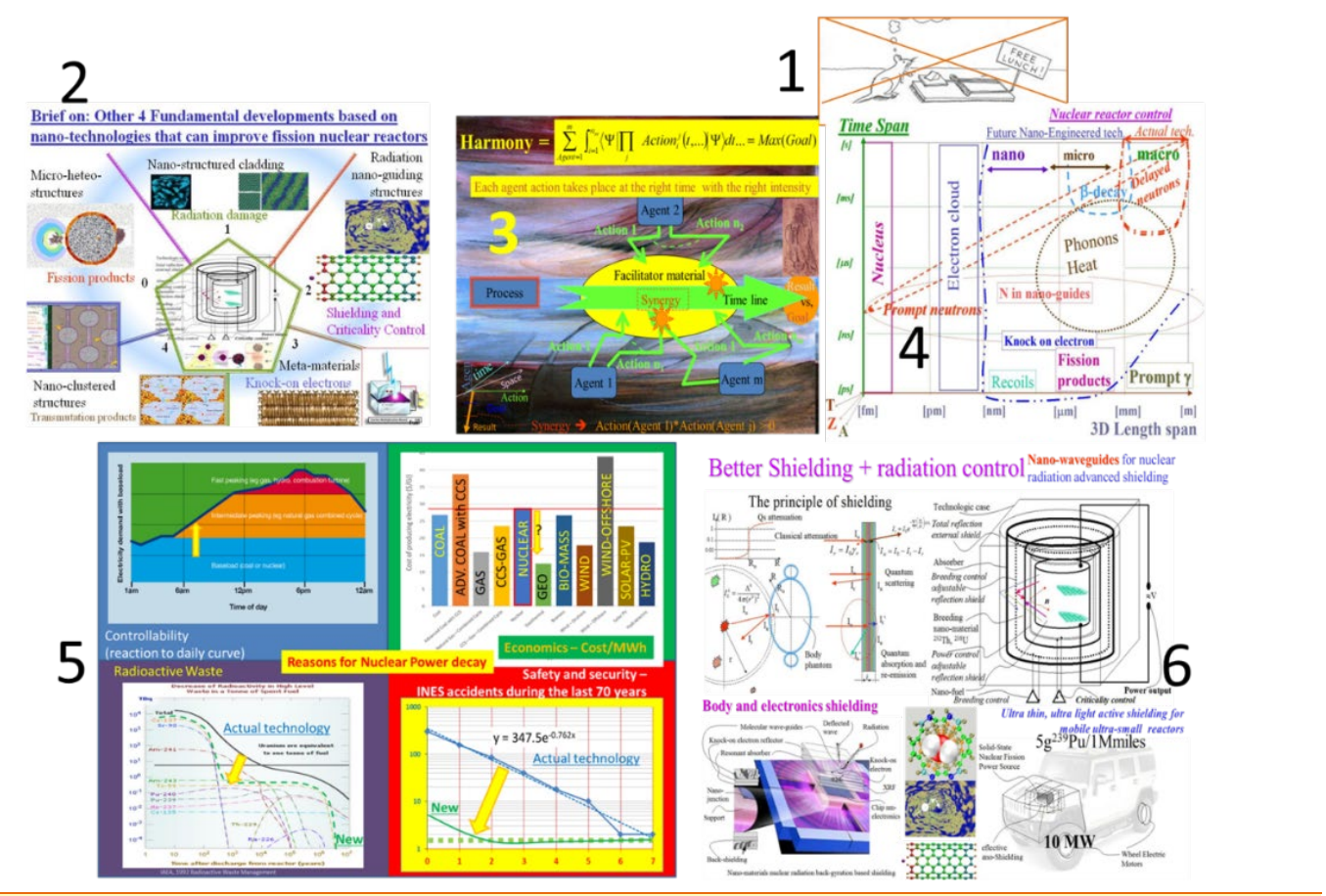

Figure 13 The 5 families of hetero-nano-structures for energy related applications

In Figure 13 top-centre " 3 " is presented the concept of harmony and synergy, where in harmony the state function gets a local extreme, and when synergy appears between 2 orthogonal processes in space the non-diagonal terms are not zero. A simple example is: $1+1=\mathrm{R}>2$ where $\mathrm{R}-2=$ Synergistic contribution. The heteromaterial represented here are mainly for fission nuclear reactor " 2 ", and are 
designed to deal and optimize the effects of major actors in the process as FPs; Knock-on electrons; Recoils; prompt neutrons; Transmutation products that may be used together or separately creating various advanced generations of nuclear reactors from $\mathrm{V}$ to VIII, ending in about 80 years with compact solid state fission battery in centre. The top-right ideogram shows that "there is no free lunch", and in spite of very nice and coherent macro-description that have been made, the devil lurks in details. What was presents are "generic" families of materials, but to build them one needs to rely on much more knowledge. The chart "4" shows the realm of each agent in a multi-scale multi-dimension (space-time) realm. The image " 5 ", intends to say that without solving at least these 4 presented might be NO "nuclear renaissance", and the applications in ideogram " 6 " are doing just that.

It is known that nuclear energy has low carbon dioxide emission, if one ignores the fuel cycle and nuclear waste "legacy", but not this technology which belongs to infancy of the nuclear power based on "hot-rod" technology to replace a fossil fuel burner with a nuclear heat source, able to produce vapours and pressure in a boiler, and use the same thermos-mechanic-electric cycle to produce and deliver electricity. For this fissile fuel is much diluted in fertile fuel, with allow burnup, and is immobilizing a considerable amount of useful fuel, generating depositing problems Popa-Simil (2012).

In Figure $14 \mathrm{AB}$ there are presented potential achievements from quantum active environments fusion/Transmutation battery.

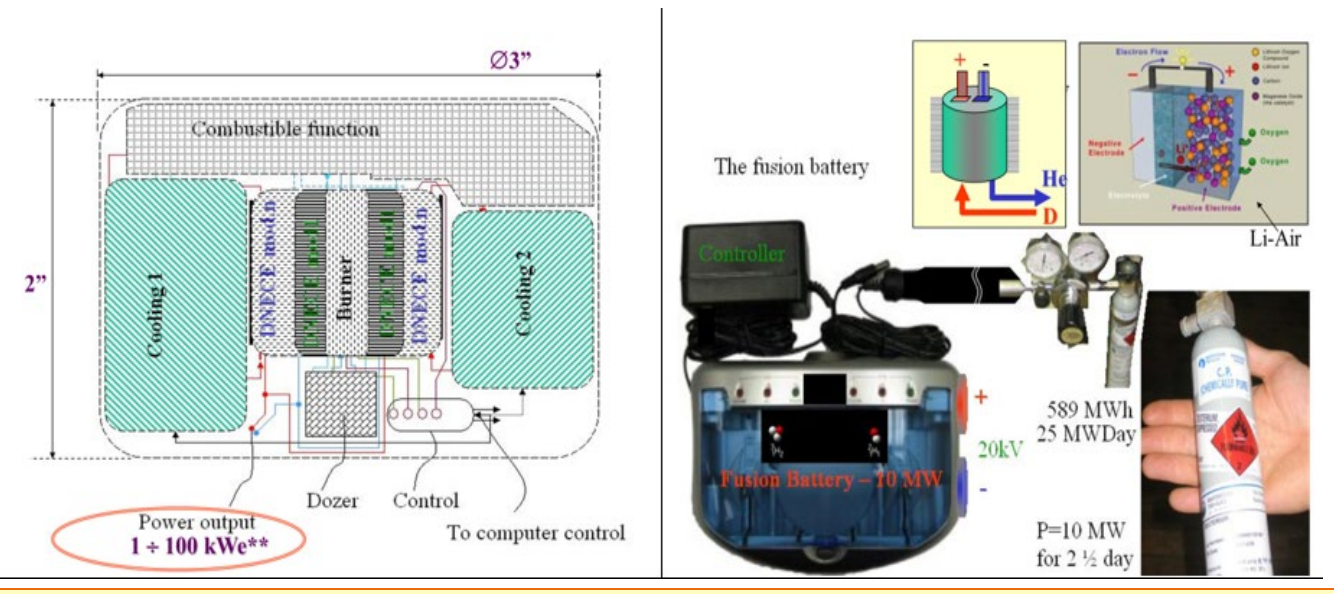

Figure 14A Fusion battery Schematic diagram

Figure 14B Example of operation

The 6th family of engineered nano-hetero structures might have the largest impact on planetary energy, is suitable for large scale multiplication and if operating the envisioned way it will make the power production portable, chemical pollution free which will allow us to increase the energy production on Earth by 2 orders of magnitude, from where energy pollution with low grade thermal will be the absolute limit, and that is about $1 \mathrm{PW}$, which will represent $0.1 \%$ of solarisation.

For the moment this development is in $\mathrm{TRL}=2$, and more research is needed until it will become a mature technology in about 100 years. By removing the chemical pollution associated with energy production this will allow an increase in planetary power by 2 orders of magnitude.

\subsection{COMPACT, SOLID-STATE FISSION BATTERY}

The 5 materials families may be used in the construction of this Generation 8 (VIII) nuclear reactor, generically called a fission battery, because uses fission 
products energy to generate electric power at will, as a difference from isotopic batteries which once loaded, they deliver continuously power.

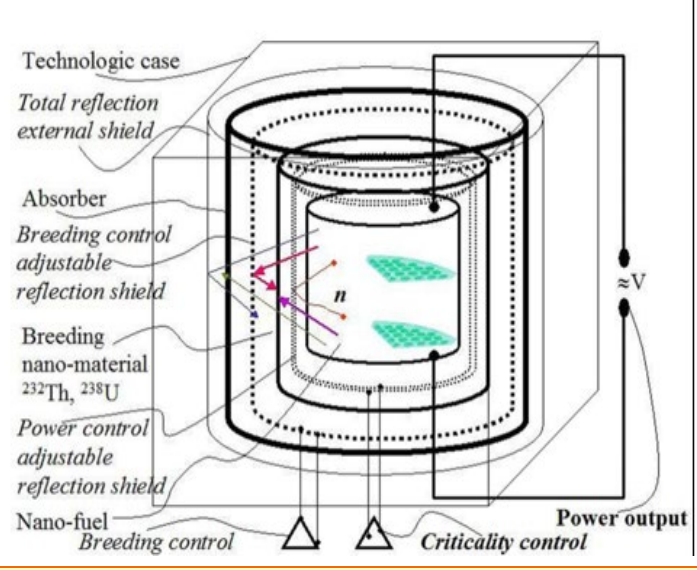

Figure 15A Fission Battery schematic design

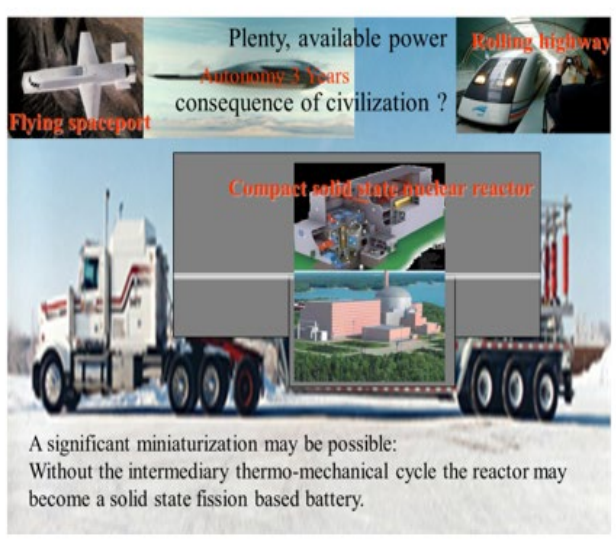

Figure 15B Fission battery applications

In Figure 15A is presented a schematic showing the inside structure of such portable battery. The present simulations showed that producing $1 \mathrm{~m} 3$ of nanohetero structures, using high enriched material is possible to reach criticality in less than $1 \mathrm{~m}$ diameter. Inside will be a hetero-structure made of DNECE structures embedded inside a Micro-Hetero structure, sunk in a drain liquid, forming the reactor's core. The efficiency is limited to about 70\%, because the nuclear recoil energy deposition range is ended in drain fluid, to increase operation lifetime and collect and extract the fission products.

On the boundaries and inside there will be active NEMS n-radiation guides, that will turn $n$ back to the active zone to increase reactivity when control voltage is on, or will let them pass out on off or Wang SanBing and ChaoHui (2017), overheating, as a failsafe mechanism, with the shutdown temperature set as function of constructive structure. Following this reflective blanket is a fertile isotope blanket using nano-clustered hetero-structure to produce isotopes of interest or for fuel breeding. Next will be a passive nano-guiding blanket, which sends all radiation towards a radiation dumper and the structure is shielded in a thermal blanket, with capabilities of switching on air cooling or liquid cooling. For amorphous nanobeaded structure operation temperature may be as high as $1200 \mathrm{~K}$, with an extraction power for cooling circuit of about $1 \mathrm{~kW} / \mathrm{cm} 3$ the reactor delivering about $2.5 \mathrm{~kW} / \mathrm{cm} 3$ where $60 \%$ directly as electricity and using a mixed cycle for electric power production, where the residual energy may be about $10 \%$, finally delivering $90 \%$ efficiency. Other designs with efficiency over $90 \%$ may be conceived for space and underwater applications, having a shorter operation lifetime, but at some of them active laser beam energy deliver may be set in place, able to produce beams of few MW power in IR/Vis. Suitable for directed energy applications in space or underwater visualization.

Figure 15B shows the level of compaction that it may be reached, having few GW power plants assembled on a trailer, and making reactors to power large airplanes, trains, ships or submarines.

\subsection{ECOLOGIC IMPACT OF THE FUEL CYCLE ENHANCEMENT}

The "Cer-Liq-Mesh" fuel and the Fractal self-repairing from radiation damage materials have a direct impact on fuel cycle, which if not smartly contained may be destructive, and detrimental, by shutting down the mining for new Uranium for 
about 30-50 years, by resuming the novel nuclear structure use the reserves already mined, and even using Depleted Uranium (DU) that remained in large amounts at enrichment plants as Y-12, LaHague, Mayak, etc. The mining reduction has to be gradual, as eliminate obsolete equipment and maintain the best and brightest. On the other hand, using stored waste fuel, in reprocessing plants will give the needed abundance of nuclear materials to still maintain at work and reprocess the actual used fuel "legacy".

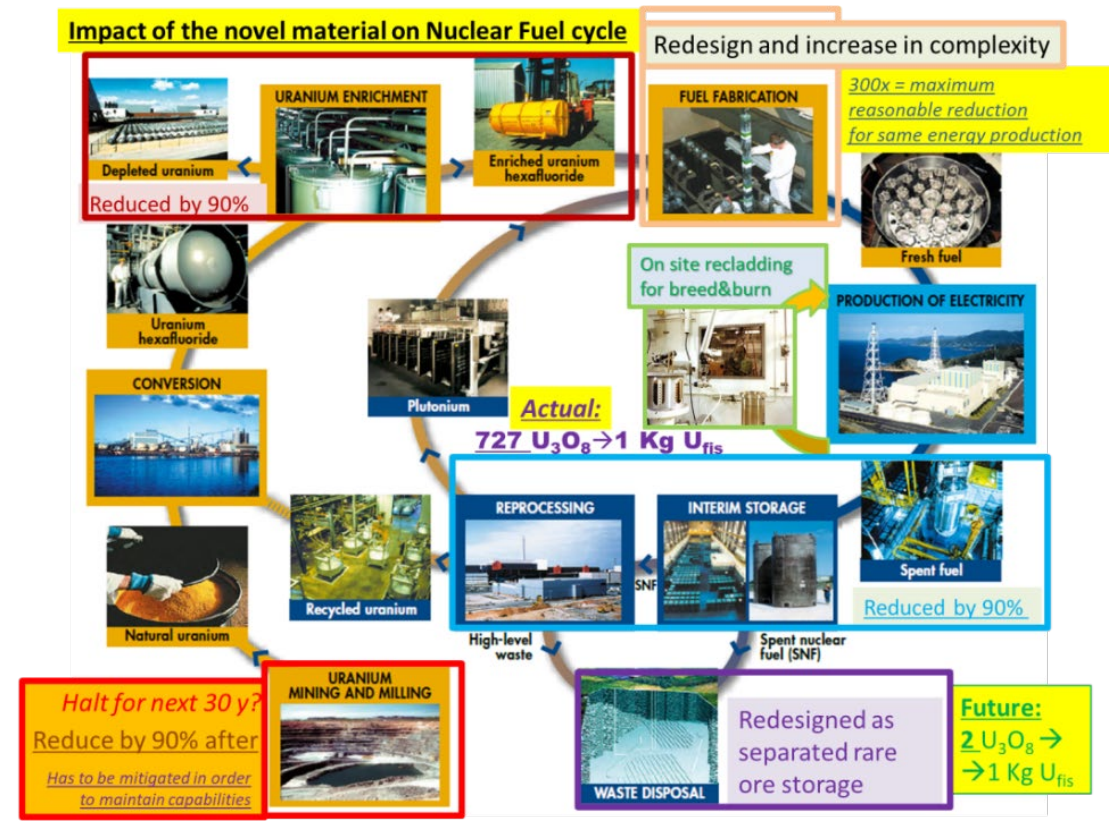

Figure 16 Fuel Cycle modifications due to novel engineered nuclear fuel

As Figure 16 shows in the bottom-left side, if not overseen a disaster similar to coal industry may happen in U mining, driving to bankruptcies and unemployment that has to be avoided by states intervention. Because the new "Cer-Liq-Mesh", is repressible on site, by light distillation and partitioning the need for enriched fuel will drastically decrease, and here is another branch of the nuclear industry which will need supervision, protection and support from states. The same will happen with the nuclear fuel reprocessing sites that will have to be tasked with the actual waste fuel reprocessing, and gradually shut down as the piles are eliminated and cleanup process is finished.

The capability offered for sealed pellets for easy recladding and fuel reprocessing and recovering by compression, up to $80 \%$ burnup when requires a Purex to be recovered and re-burned saves environment more than $80 \%$ from the actual exposure to harsh chemicals and extensive nuclear materials processing waste. The on-site reprocessing eliminates proliferation thread, simplifying security measures, while for each GWDay of energy produced at maturation only $2 \mathrm{~kg}$ of fission products have to be appropriately stored, not as waste but as future rare ore, reducing the actual volumes by up to 5 orders of magnitude, that will gradually improve. 


\subsection{STRATEGIC IMPORTANCE OF NOVEL NUCLEAR POWER SOURCES}

Figure 17 is presenting another application very important for environment that is suppressing the wars for planetary resources and local hegemony, and moving forward towards an era of negociations under UN umbrella.

\section{Wars denial using advanced power sources in strategic applications BMD case}

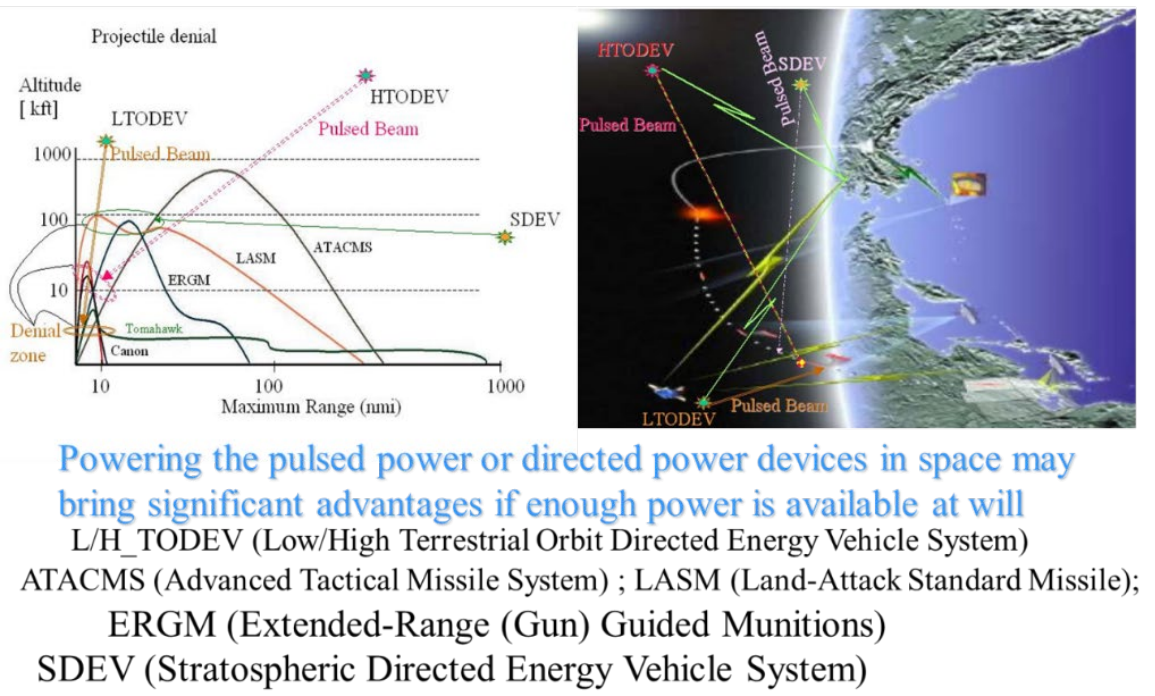

Figure 17 Applications of Nuclear batteries in space for directed energy devices

Placing in space a high efficiency nuclear battery, able to power high power lasers, or accelerator beams into desired targets may be used to clean up the outer space from space-junk, defend Earth against asteroid collisions or serving as directed energy resource in LTO (Low Terrestrial Orbit) to deny wars by denying air-space and even artillery shells and incapacitating aggressive military resources on ground. Everything in space is about high efficiency and as low as reasonably possible power. Using supercapacitors formed of hyperbolic meta-structures the converted energy may be directly extracted as an ultra-high power laser beam, directed on space targets or as an illumination tool for under-water visualization. It may also power high power phased arrays for underground down to $1 \mathrm{~km}$ or more visualization of geological features or DBTs (Deep Buried Targets) bringing important strategic advantages, or simply submarines and ships and other objectives in artic climate. In case of weather challenges, it will be the easiest deployable power source.

\section{CONCLUSIONS}

As one can see in Figure 18B, about the oldest known nano-material mankind unconsciously produced, the Damascus steel, which dictated over exceptional performances of Damascus swords, so in the actual technology the nuclear engineered nano-materials will finally determine the performances of the energy related devices they were made of. 


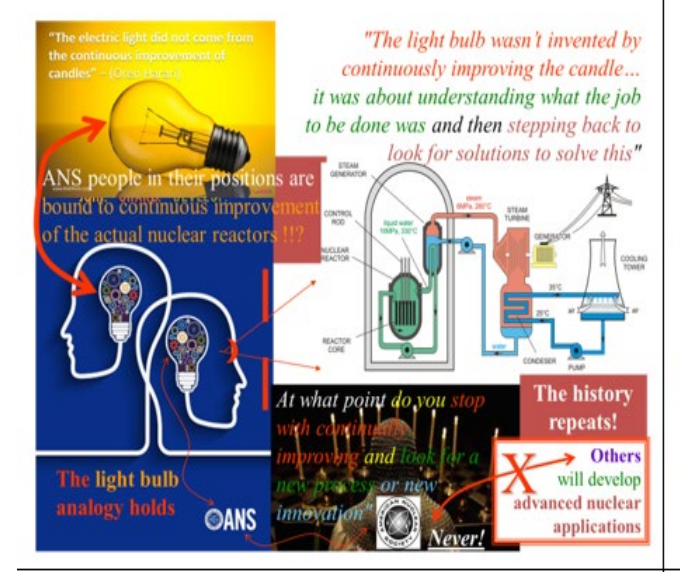

Figure 18 A Nuclear Inventions and innovation

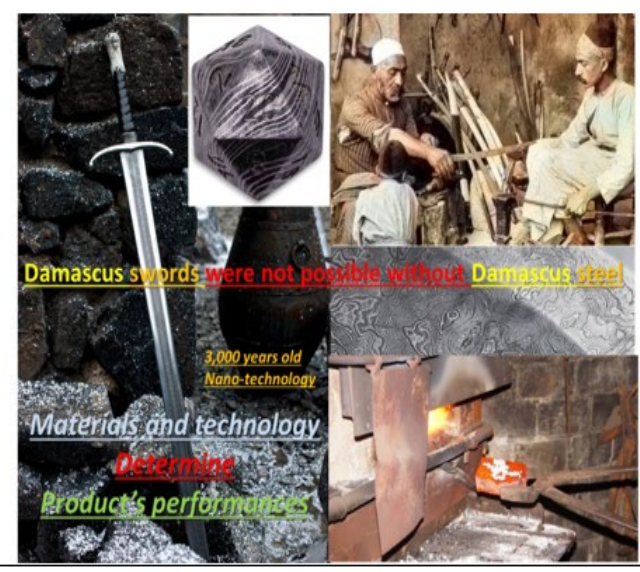

Figure 18 B Damascus steel vs sword quality

Figure 18A, shows the analogy between nuclear reactor progress and development of Electric light citing Oren Harari. In fact, nuclear guild is among the most conservative in the world, and now they are still improving 1950 technology, which as candles in 1800 were a mature technology, for which improvements have less and less qualitative effects, and new brains were needed to develop electric light based on hot $\mathrm{W}$ wires in vacuum or most recently high efficiency LEDs.

The technologies presented have 40 years of continuous smooth development, and without as serious consideration from a smart state, it will may take another 100 years until reaching TRL=10 and another century to maturation, and probably it will be too little too late, for a population terminated by climate challenge and social unrest triggered by scarce resources from a distressed environment.

The impact on world these systems may bring is great, as with enough energy all other resources may be had, and nuclear power may become one of the most environmentally and man friendly power supplies, because mankind will badly need energy when the weather is bad, and renewable sources do not deliver as needed. The development of the 6th family of materials will drive to scientific leap making us understand more about quantum features of nuclear forces, as well about teleportation, entanglement and universe, helping us conceive and invent many other technologies.

\section{REFERENCES}

Estrin Y., K.H.S., Nabarro F.R.N., (2007) "A comment on the role of Frank-Read sources in plasticity of nanomaterials". Acta Materiala. 55: p. 6401-6407. Retrieved from https://doi.org/10.1016/j.actamat.2007.07.052

Keller John, (2018) "Directed-energy weapons like laser weapons, microwaves, and particle beam weapons are future of defence", Military \& Aerospace Electronics,

Meliopoulos Sakis, T.D., Singh Chanan, Yang Fang, Kang Sun Wook, Stefopoulos George,, (2005) "Comprehensive Power System Reliability Assessment". Power Systems Engineering Research Center. PSERC Publication 05-13.

Nabarro F.R.N., (1998) "Stress-Driven Grain Growth", Scripta Materialia. 39(12)): p. 1681-1683. Retrieved from https://doi.org/10.1016/S13596462(98)00350-9

Olander D.R., (1976) "Fundamental Aspects of Nuclear Reactor Fuel Elements", TID26711-P1. Retrieved from https://doi.org/10.2172/7343826 
Ponomarev-Stepnoi N.N., Usov V.A.,, (2000) Russian space nuclear power and nuclear thermal propulsion system",. Nuclear News. 43(13): p. 33-46.

Popa Simil Liviu, (2018) Micro-Nano hydraulics in hetero-structures developed for nuclear applications. J Pharm Sci Emerg Drugs. 6. Retrieved from https://doi.org/10.4172/2380-9477-C6-021

Popa-Simil L., (2007) "Nano-structures materials for Energy Direct Conversion and Fuel Breeding", Nanotech 2007. 4(7): p. 679 - 682.

Popa-Simil L., (2004) "Report on Nuclear Fuel's Thermal Conductivity Modeling and Measurement - Urania". LANL Reports. LA-UR-04-5194(1): p. 1-45.

Popa-Simil Liviu, (2017) Accelerator Enabled Nano-Nuclear Materials Development. Advanced Nano-Energy. 1(1): p. 12.

Popa-Simil Liviu, (2012) Applied Nano-technologies Improves Nuclear Power Safety and Performances. Kindle Edition. Nano-technologies in Nuclear Power Book 1: p. 429

Popa-Simil Liviu, (2018) "Nano-Engineered nano-nuclear materials susceptible to trigger a leap forward in planetary civilization". Nano S\&T. Germany.

Popa-Simil, L., (2015) "Accelerated Discovery and Design of Nano-Material Applications in Nuclear Power by Using High Performance Scientific Computing",Research and Applications in Global Supercomputing, Ch. 4-5, 83-148. Retrieved from https://doi.org/10.4018/978-1-4666-74615.ch004

Popa-Simil, L., (2007) "Micro-structured nuclear fuel and novel nuclear reactor concepts for advanced power production", Progress in Nuclear Energy 50(2):539-548. Retrieved from https://doi.org/10.1016/j.pnucene.2007.11.041

Saarenvirta Gary, (2009) "Disruptive Innovation versus Continuous Improvement", The Power of Innovation

Schmidt George, H.M., (2006) Radioisotope-based Nuclear Power Strategy for Exploration Systems Development. STAIF Nuclear Symposium : p. 813. Retrieved from https://doi.org/10.1063/1.2169210

Seligman Lara, (2016) "Lockheed's Marillyn Hewson Touts Breakthroughs in Hypersonic Weapons", Defense News.

Staub D. W., (1967) SNAP 10A "Summary Report". Atomics International Report NAA-SR-12073.

Ursu I., P. I. I. (1980). "Synergetics of the fission electric cells." International Journal of Energy Research 4(1): 19-30. Retrieved from https://doi.org/10.1002/er.4440040104

Ursu I., P. I., Badescu-Singureanu A., Vamanu D., (1978) "Cell for obtaining electric energy directly from the nuclear fission energy". Romania, Institute for Physics and Nuclear Energy: 4.

Wang SanBing, X.Q., He ChaoHui,, (2017) Application of the Burnable Poison in the Design of Space Nuclear Reactor. 25th International Conference on Nuclear Engineering. 3 - Nuclear Fuel and Material, Reactor Physics and Transport Theory; Innovative Nuclear Power Plant Design and New Technology Application; Shanghai, China, July 2-6: p. 5. Retrieved from https://doi.org/10.1115/ICONE25-66393

Weber C.E., H.h., (1956) "Dispersion-Type Fuel Elements". International Conference on the Peaceful Uses of Atomic Energy. 9,(United Nations): p. 196. 